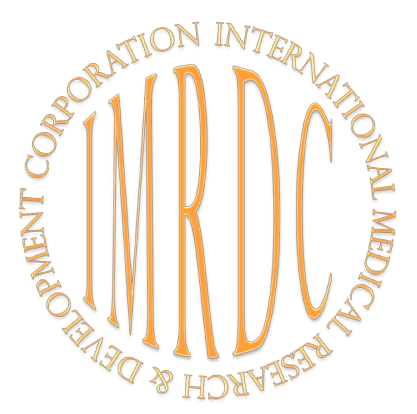

\title{
Large Fungal Ball of the Paranasal Sinuses and Nasal Cavity: Two Case Reports
}

\author{
Dmitrii A. Shcherbakov, $\mathrm{PhD}, \mathrm{ScD}^{1}$; Natalya V. Klimova, $\mathrm{PhD}, \mathrm{ScD}^{2}$; \\ Tatyana Yu. Malysheva ${ }^{3}$; Augul F. Shcherbakova ${ }^{1}$ \\ ${ }^{1}$ Tyumen State Medical University, Tyumen, Russia \\ ${ }^{2}$ Surgut State University, Surgut, Russia \\ ${ }^{3}$ Tyumen Regional Hospital, Tyumen, Russia
}

\begin{abstract}
Two cases of a large fungal ball of the paranasal sinuses and nasal cavity are presented and its removal via an endoscopic approach is detailed. The clinical symptoms of the fungal body of the paranasal sinuses are not specific. With one-sided localization and large sizes of fungal bodies, they must be differentiated from neoplasms. The most informative non-invasive diagnostic method is computed tomography. We are presenting two cases of giant fungal bodies of the paranasal sinuses and identified specific CT signs. (International Journal of Biomedicine. 2019;9(4):376-378.)
\end{abstract}

Key Words: fungal ball $\bullet$ nose neoplasms $\bullet$ endoscopic sinus surgery

\section{Introduction}

Fungal balls of the paranasal sinuses are a non-invasive form of fungal rhinosinusitis, characterized mainly by a unilateral lesion, without involving the underlying mucosa in the inflammatory process. ${ }^{(1)}$ The main clinical manifestations, such as nasal congestion, mucopurulent discharge or bleeding, and headache, are not specific and are found in many diseases of paranasal sinuses..$^{(2)}$

The most informative non-invasive diagnostic methods are computed tomography (CT) and magnetic resonance imaging (MRI). According to the results of $\mathrm{CT}$ of the paranasal sinuses, it is possible to suspect the presence of a fungal ball in cases of total or subtotal darkening of the paranasal sinus with calcinates. In cases of long-term current processes, due to compression of the walls of the paranasal sinus on CT images, erosion of the paranasal sinus wall can be detected in about 3.6\%-17\% of cases. ${ }^{(3,4)} \mathrm{An}$ MRI is indicated for pregnant patients in cases of long-term, unilateral sinusitis. ${ }^{(5)}$ On an MRI of paranasal sinuses, FBs are visualized on T1-weighted

*Corresponding author: Prof. Dmitrii A. Shcherbakov, PhD ScD. Tyumen State Medical University, Tyumen, Russia. E-mail: dmst@bk.ru images in the form of areas with low signal intensity, and on $\mathrm{T} 2-\mathrm{VI}$ in the form of areas with a hypointense signal or parts of the "empty" signal.

\section{Case Report 1}

A 59-year-old white male presented to the otorhinolaryngology department. During the previous four years, manifestations of chronic right-sided rhinosinusitis had arisen. Two months after the patient first presented to the otorhinolaryngology department, the condition worsened, and the patient sought medical help in the outpatient clinic with complaints of painful pressure in the right half of the face, difficulty with nasal breathing on the same side, mucopurulent discharge on the right, and intoxication syndrome. According to the endoscopic examination of the nasal cavity after mucous membrane anemization, we revealed: in the nasal cavity on the right, the polyposis, a mucopurulent discharge, and the structures of the nasal cavity were not differentiated.

A CT scan was obtained showing a soft tissue density in the nasal cavity on both sides, the right maxillary sinus and cells of the ethmoid bone on the right, the bone structures of the right orbit were preserved and the nasal septum was displaced to the left (Fig.1). With the patient under general anesthesia, we 
performed infiltration with articaine $+1: 200000$ adrenaline of the anterior end of the inferior nasal concha and lateralization of the inferior nasal concha, and we visualized the caseous mass (Fig.2) occupying the nasal cavity, right maxillary sinus and the right ethmoid labyrinth. After removing the mass, a single cavity of the right maxillary sinus and the right ethmoid labyrinth was determined. This cavity was washed with $0.9 \%$ saline solution. According to histological data, the mass was determined to be fungal flora of Candida albicans. On the control CT scan one week after the surgical intervention, we revealed a single cavity of the maxillary sinus and an ethmoid labyrinth on the right (Fig.3).
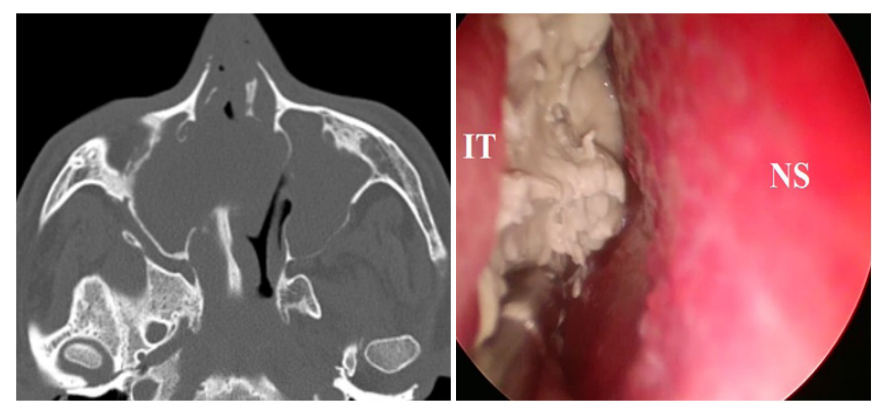

Fig. 1. A 59-year-old white male. Fig. 2. Intraoperative photo: caseAxial CT scan at the level of the ous masses occupying the right lesion. The isodensive shading on nasal cavity.

the right side, involving maxillary Fungal flora: Candida albicans. sinus and the ethmoid cells. The IT-inferior turbinate, NS-nasal nasal septum is displaced to the left. septum.

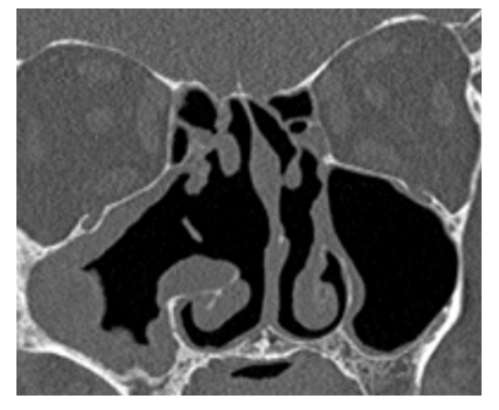

Fig, 3. The control CT scan one week after the surgical intervention. A single cavity of the maxillary sinus and an ethmoid labyrinth on the right.

\section{Case Report 2}

A 40-year-old white male presented to the otorhinolaryngology department of Tyumen Railway hospital. When admitted, he complained about the absence of nasal breathing through the right half of the nose, nasal discharge, a putrid odor from the nose, and a high-grade body temperature for 6 months. According to his medical history, since June 2018 there had been a difficulty with nasal breathing on the right, anosmia, and subfebrile body temperature. He was treated in the outpatient clinic, and underwent several courses of antibacterial therapy; the patient regularly used vasoconstrictive drops without positive dynamics. About 3-4 years ago, he had treatment for the teeth in the upper jaw on the right; there were no complications after the treatment. Patient had viral hepatitis C. A CT scan of the paranasal sinuses was performed and the patient was referred to the otorhinolaryngologic department with a diagnosis of paranasal sinus neoplasm.

According to the endoscopic examination of the nasal cavity after mucous membrane anemization, in the nasal cavity on the right the polyposis and a mucopurulent discharge was presented, and the structures of the nasal cavity were not differentiated. The nasal septum was sharply displaced to the left. The CT scan (Fig.4) showed destruction of the medial wall of the right maxillary sinus and of the structures of the ethmoid labyrinth on the right, and displacement of the median structures sharply to the left. Bone structures of the right orbit were preserved. Under general anesthesia, the right-sided FESS was performed. The fungal ball was fragmented and removed (Fig.5).

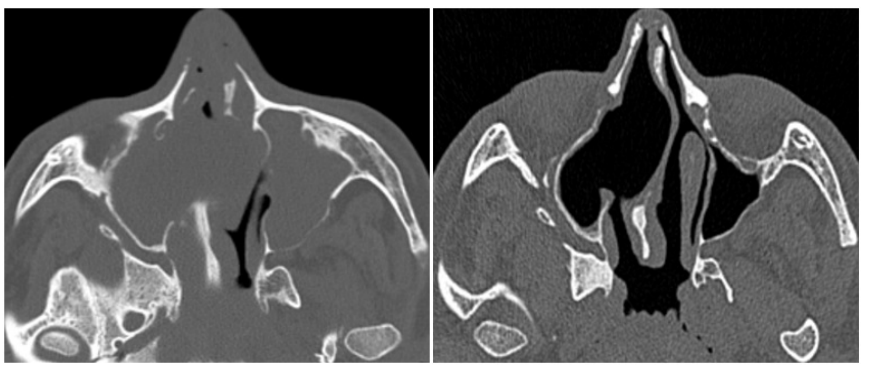

Fig. 4. A 40-year-old white male. Fig. 5. The control CT scan one Coronal CT scan at the level of week after the surgical interthe lesion. Destruction of the vention. A single cavity of the medial wall of the right maxillary maxillary sinus and an ethmoid sinus and of the structures of the labyrinth on the right. ethmoid labyrinth on the right, and displacement of the median structures sharply to the left.

\section{Discussion}

In recent years, the incidence of fungal rhinosinusitis has grown. Owing to imaging and diagnostic endoscopy, it is possible to define the classification, diagnosis and management of these diseases more precisely. Fungal rhinosinusitis is generally classified into invasive and non-invasive.

The non-invasive form, in its turn, is subdivided into allergic fungal sinusitis and fungal ball. The first to describe a case of a non-invasive fungal sinusitis was probably Mackenzie in 1893. ${ }^{(6)}$

The fungal ball (mycetoma) can be described as an accumulation of non-invasive dense fungal concretions at the level of the paranasal cavities. It is more often unilateral although rarely it can affect more sinus cavities. The disease generally occurs in adults, with a mean age of 64 years, predominantly among women. However, we have now presented two cases of a large fungal ball of the paranasal sinuses and nasal cavity in two male patients aged 40 years old and 59 years old.

The fungal ball differs from allergic fungal sinusitis, which generally affects more sinuses. The fungal ball is most often localized at the level of the maxillary sinus. The opacification of the sphenoid sinus is very rare, found in $5 \%$ of patients with diseases of the paranasal sinuses, of which only $5 \%$ are suffering from fungal ball. ${ }^{(7)}$ 
Patients with fungal ball are almost all immunocompetent, without significant alterations in the levels of immunoglobulin or IgG subclasses. Because of its slow evolution, few symptoms, and non-invasiveness, the diagnosis of paranasal sinus fungal ball is often late. Generally, the symptoms include headache, nasal breathing discharge, and facial algia and are often similar to symptoms of bacterial chronic rhinosinusitis.

CT findings associated with the presence of paranasal sinus fungal ball are characterized by the presence of inhomogeneous material at the level of the paranasal sinus involved, which often appears obliterated, with the presence of some material hyperdense spot or a single focus hyperdense. The sensitivity and specificity of CT, in the presence of these findings, was calculated respectively in $62 \%$ and $99 \%$. Although the CT has high sensitivity and specificity, it is always necessary to rule out other sinus disorders, benign or malignant.

We have identified distinctive CT signs for the large paranasal sinus fungal ball: the destruction of the medial wall of the paranasal (maxillary) sinus by the fungal mass, intact orbital wall, and displacement of the nasal septum.

The treatment of fungal ball can be performed through the classic surgery of Caldwell-Luc, both using endoscopic techniques as it was in our case. The result is excellent with both techniques and rarely requires a systemic antifungal therapy.

\section{Competing Interests}

The authors declare that they have no competing interests.

\section{References}

1. Montone KT. Pathology of Fungal Rhinosinusitis: A Review. Head Neck Pathol. 2016;10(1):40-46. doi: 10.1007/ s12105-016-0690-0.

2. Hoggard M, Wagner Mackenzie B, Jain R, Taylor MW, Biswas K, Douglas RG. Chronic Rhinosinusitis and the Evolving Understanding of Microbial Ecology in Chronic Inflammatory Mucosal Disease. Clin Microbiol Rev. 2017;30(1):321-348.

3. Zhu H, Zhang W, Guan J, Ye H, Su K. CT imaging and clinical features of sinus fungus ball with bone erosion. $\mathrm{J}$ Nature Science. 2015;1(4):e69.

4. Eloy P, Marlair C, de Dorlodot CL, Weynand B Maxillary and Sphenoid Sinus Fungus Ball: A Single Belgian Centre's Experience. J Otol Rhinol.2014;3:6.

5. Scherbakov DA, Malysheva TYu, Adamchuk AM, Ekimova AE, Krotova AS. [Application of magnetic resonance imaging in the diagnosis of fungal body in maxillary sinus. Clinical case]. Folia Otorhinolaryngologiae et Pathologiae Respiratoriae. 2017; 1(23): 74-79.[Article in Russian]. 6. Mackenzie JJ. Preliminary report on aspergillus mycosis of the antrum maxillare. John Hopkins Hosp Bull. 1893;4:9-10.

7. Dhong HJ, Jung JY, Park JH. Diagnostic accuracy in sinus fungus balls: CT scan and operative findings. Am J Rhinol. 2000;14(4):227-31. 